Cahiers de philosophie de l'université de

\title{
Simon Frank lecteur de Plotin
}

Jérôme Laurent

\section{(2) OpenEdition}

Journals

Édition électronique

URL : https://journals.openedition.org/cpuc/998

DOI : 10.4000/cpuc.998

ISSN : 2677-6529

\section{Éditeur}

Presses universitaires de Caen

\section{Édition imprimée}

Date de publication : 31 octobre 2011

Pagination : 135-149

ISBN : 978-2-84133-385-1

ISSN : 1282-6545

Référence électronique

Jérôme Laurent, « Simon Frank lecteur de Plotin », Cahiers de philosophie de l'université de Caen [En ligne], 48 | 2011, mis en ligne le 01 septembre 2020, consulté le 10 février 2023. URL : http:// journals.openedition.org/cpuc/998; DOI : https://doi.org/10.4000/cpuc.998

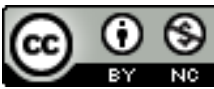

Creative Commons - Attribution - Pas d'Utilisation Commerciale 4.0 International - CC BY-NC 4.0 https://creativecommons.org/licenses/by-nc/4.0/ 


\section{Simon Frank lecteur de Plotin}

Gimon Frank est l'une des figures les plus éminentes de la philosophie $\mathcal{O}$ russe au XX ${ }^{\mathrm{e}}$ siècle; sa thèse de doctorat éditée à Petrograd en 1915 fut partiellement traduite en français et parut en 1937 dans la collection "philosophie de l'esprit» chez Aubier-Montaigne. Louis Lavelle et René Le Senne qui présentaient l'ouvrage et l'auteur dans leur collection en faisaient un partisan de la «mystique spéculative» issue de Plotin et de Nicolas de Cuse, proche du (néo)-platonisme d'un Bradley. Ils justifiaient le projet dans une opposition frontale au "positivisme» du XIX ${ }^{e}$ siècle dans une perspective "spiritualiste» que nos jeunes lecteurs auront peut-être du mal à imaginer tant l'expression "philosophie de l'esprit» a changé de signification en ce début de $\mathrm{XXI}^{\mathrm{e}}$ siècle:

À l'encontre du matérialisme, qui ontologise le non-esprit, et du positivisme, assez naïf pour croire que l'utilité et le plaisir suffisent à l'homme et assez partial pour considérer comme une maladie mentale tout effort vers l'Absolu, se définissent des doctrines qui, sous des noms différents, mais par des voies convergentes, reviennent au Principe réel qui nous illumine et nous inspire, si seulement nous ne nous détournons pas de lui par le refus de nous associer à sa bienfaisance ${ }^{1}$.

Si de nos jours la philosophie se divise grosso modo en trois branches - la philosophie "analytique», la phénoménologie et le rationalisme issu de Kant -, à l'époque de Lavelle, avant la Seconde Guerre mondiale, la division était binaire: Spiritualistes contre Matérialistes. Le succès de Sartre, puis l'influence croissante des «maitres du soupçon» - Freud, Nietzsche et Marx -, l'apparition du structuralisme dans les années soixante, tout cela mit fin à cette dualité et mit au placard, si l'on ose dire, les tenants du spiritualisme, dont l'œuvre de Bergson qui connut un long «purgatoire»

1. L. Lavelle, R. Le Senne, in S. Frank, La connaissance et l'être, Paris, Aubier-Montaigne, 1937 (trad. du russe par Kaffi, Oldenbourg et Fedotoff), p. III. Le texte russe (Предмет знания) a paru à Pétrograd en 1915 aux éditions Schreder. 
jusqu'aux travaux récents et une sorte de redécouverte de son œuvre dans les années quatre-vingt-dix. Qui se souvient du livre de Frank paru en 1937 et que citait pourtant en bonne part Étienne Gilson dans L'être et l'essence ${ }^{2}$ ? Il a fallu attendre 2007 pour que l'opus magnum, le grand livre de Frank, paru à Paris, en russe, en 1939, L'inconcevable, soit traduit en français ${ }^{3}$ !

L'œuvre de Frank a une force singulière qui en fait l'un des rares néoplatoniciens chrétiens du $\mathrm{XX}^{\mathrm{e}}$, mais un néoplatonicien imprégné de la philosophie post-kantienne et de la phénoménologie. Les spécialistes de Husserl retrouveront bien des analyses des Ideen reprises par Frank, orientées vers un sens et un horizon nouveaux.

Comme le note Pierre Caussat dans sa Présentation à L'inconcevable:

C’est Plotin qui demeure la référence majeure, la source vive où s'alimente la spéculation de Frank. Illustration éclatante: presque tous les chapitres de L'objet du savoir [ = La connaissance et l'être] portent en épigraphe une citation des Ennéades. [...] Frank, ou la version rénovée, revisitée du plotinisme 4 .

J'aimerais dans un premier temps indiquer l'ensemble des citations de Plotin que Frank a mises en exergue de certains chapitres de La connaissance et l'être, citations qui ne figurent pas toujours dans la traduction partielle du livre en 1937.

\section{Dans La connaissance et l'être:}

Au chapitre IV, «La conscience et l'être. La conscience de l'être absolu », dans l'édition russe, on trouve la citation suivante (en grec) que je donne en français, dans la traduction des Ennéades par Émile Bréhier, comme pour les autres citations mises en exergue:

Cette multiplicité réelle [l'Intelligence divine], c'est l'intelligence et l'intelligible pris ensemble (ä $\mu$ / hama), et les deux à la fois (hama); ils sont deux; il faut donc un principe antérieur au couple. [...] S'il n'est ni l'intelligence ni l'intelligible, qu'est-il donc? - Celui d'où vient l'intelligence et, avec elle, l'intelligible, répondrons-nous. [...] Par quelle sorte d'impression pouvons-nous saisir d'un

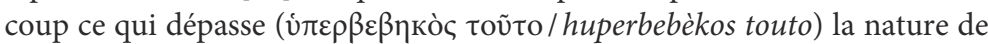
l'intelligence? - Expliquons-le, autant qu'il est possible: par ce qui, en nous, est semblable à ce principe, répondrons-nous 5 .

2. É. Gilson, L'être et l'essence, Paris, J. Vrin, 1948, p. 285.

3. S. Frank, L'inconcevable, Paris, Cerf et Genève, Ad Solem (La nuit surveillée), 2007 (trad. par P. Caussat, avec la collaboration de B. Marchadier).

4. P. Caussat, Présentation à S. Frank, L'inconcevable, p. 22 et 23.

5. Plotin, traité Sur la contemplation, III, 8 [30], 9, lignes 5-6, 11-13 et 21-23, in Ennéades (trad. par É. Bréhier). 
Et, aussitôt après :

Intelligence, intelligible et être ne font qu'un; c'est là le premier être ${ }^{6}$.

Une première remarque s'impose, toute formelle: Frank fragmente le texte qu'il cite, usant finalement de la même liberté que Plotin par rapport à Platon.

Le chapitre V, «De la nature du lien logique», commence par cette citation, cette fois présente, traduite, dans la version française:

Dans la science [...], certes, actuellement, le savant n'a en vue que la partie de la science dont il a besoin, et cette partie est mise au premier rang; mais les autres aussi la suivent, parce qu'elles y sont en puissance, de façon latente, de sorte que toute la science est dans cette partie [...]. Il ne faut pas s'imaginer qu'un théorème d'une science soit isolé des autres ${ }^{7}$.

Au chapitre VI, «L'Un (Alleinheit) et la loi de détermination», nous trouvons cette citation en exergue:

Nous possédons les idées de deux manières: dans l'âme, elles sont développées et [comme] séparées l'une de l'autre; dans l'intelligence, elles sont toutes

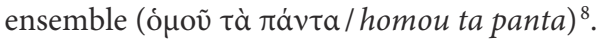

Au chapitre VII, «La première détermination et la nature de l'intuition » :

Il faut $[. .$.$] entendre par intelligence autre chose que la faculté de raisonner et$ de conclure (logistikos); [...] il faut dire que l'intelligence [...] contient tout en elle-même et qu'elle est toutes choses, multiplicité indivise et pourtant distincte; la distinction n'y est pas celle des paroles qui sont pensées une à une; et pourtant il n'y a pas de confusion entre ses parties; chacune ressort séparée des autres; il en est comme dans les sciences; tout ce que l'on sait est indivisible et pourtant tout est séparé de tout ${ }^{9}$.

Au chapitre X, «Le temps et le nombre» :

Dire que le temps est la vie de l'âme consistant dans le mouvement par lequel l'âme passe d'un état de vie à un autre état de vie, ne serait-ce pas dire quelque chose $^{10}$ ?

6. Plotin, traité Sur les hypostases qui connaissent, V, 5 [49], 5, 26-27, ibid.

7. Plotin, traité Sur l'unité des âmes, IV, 9 [8], 5, 13-15 et 19-20, ibid.

8. Plotin, traité Qu'est-ce qu'un vivant?, I, 1 [53], 8, 6-8, ibid.

9. Plotin, traité Du bien ou de l'Un, VI, 9 [9], 5, ibid.; la traduction française qui figure en 1937 est sans doute due aux traducteurs russes: «Il faut considérer la raison en tant qu'elle est autre que la raison discursive ou, comme on l'appelle, logique; c'est la raison en tant qu'immobile, qui contient toutes choses en soi et est toutes choses, une totalité indivise et aussi subdivisée, de sorte que les notions y sont unifiées sans y être confondues; car chacune s'y trouve à part, comme dans les sciences où chacune est distincte dans le tout du savoir" (La connaissance et l'être, p. 180); la traduction Bréhier est plus exacte.

10. Plotin, traité De l'éternité et du temps, III, 7 [45], 11, 43-45, ibid. 
Et enfin au chapitre XII, «L'être concret et la connaissance vivante»:

Cette vie, c'est la sagesse (hè de zồ sophia), une sagesse qui ne s'acquiert pas par la réflexion (logismois), parce que toujours elle est là tout entière, sans une défaillance, qui seule exigerait la recherche réfléchie (zètèsis): elle est la sagesse première, qui ne vient pas d'une autre; c'est l'être même qui est la sagesse (hè ousia autè sophia) ${ }^{11}$.

L'ensemble de ces citations converge vers l'idée fondamentale du premier livre de Frank: «l'objet du savoir» - «Предмет знания » - est un objet total, une «matière» que l'on pense et qui nous englobe, étant l'être lui-même. Le terme russe предмет / predmiet n'est pas littéralement «l'objet» opposé au sujet, c'est à la fois la matière d'enseignement, le sujet d'un article ou la branche d'une spécialité, bref c'est ce sur quoi porte la connaissance ou le savoir: l'intelligible.

La thèse néoplatonicienne suppose l'existence d'un monde intelligible où l'être et l'esprit sont convertibles, où l'intelligible est en même temps l'intelligence, l'Idée une activité noétique. Plotin écrit ceci:

Qu'est donc l'idée? Une intelligence ou une substance intellectuelle (nous kai noera ousia); chaque idée n'est point différente de l'Intelligence; elle est une intelligence. L'intelligence complète est faite de toutes les idées, et chacune des idées, c'est chacune des intelligences: de même la science complète est faite de tous les théorèmes, et chaque théorème est une partie de la science complète, non pas une partie localement séparée des autres, mais une partie qui a, dans l'ensemble, sa propriété particulière ${ }^{12}$.

Ce qui vaut pour une science dans notre monde sensible voué à la discursivité et à l'extraposition, vaut a fortiori pour le monde intelligible où «tout est ensemble» selon une formule souvent utilisée dans les Ennéades. Or une science est comme un système logique où les propositions (les «theorèmata» dans un sens assez général) s'impliquent mutuellement. Certes, dans l'exercice de l'homme de science tout n'est pas confondu et placé au même niveau, mais dans sa puissance spéculative, il peut actualiser à volonté telle ou telle partie («non séparée») de son savoir. Un exemple particulièrement important est celui des nombres auquel Plotin consacre

11. Plotin, traité Sur la beauté intelligible, V, 8 [31] , 4, 35-38, in Ennéades (trad. par É. Bréhier). Frank se réfère également à ce même chapitre 4 où Plotin parle de Lyncée: "On l'imagine bien [une Idée dans le monde intelligible] comme une partie, mais un regard perçant y voit le tout; comme si l'on avait une vue pareille à celle de Lyncée qui, dit-on, voyait même ce qu'il y a à l'intérieur de la terre; car cette fable nous suggère l'idée des yeux tels qu'ils sont là-bas» (lignes 23-26). Voir S. Frank, La connaissance et l'être, p. 236-237 (texte russe, p. 315).

12. Plotin, traité Sur l'Intelligence, les Idées et l'être, V, 9 [5], 8, 2-7, ibid. 
tout un traité ${ }^{13}$, et Frank tout un chapitre dans sa thèse ${ }^{14}$. Dans la tradition platonicienne, les nombres sont des réalités intelligibles qui existent «en elles-mêmes», "en soi», voulant dire par là que les nombres ne sont pas seulement des propriétés empiriques des choses, issues de l'expérience sensible. Dans le Philèbe, Platon distingue ainsi l'unité d'un vivant (l'homme ou le bœuf), où l'unité est le prédicat d'une multiplicité (un vivant est une synthèse de parties distinctes) et l'unité en tant que telle, que le grec nomme henas,-ados, en français "hénade» (comme on parlera de triade pour le trois «en soi» ou de décade pour le dix «en soi») ${ }^{15}$. Plotin distingue ainsi les nombres «nombrants» qui sont une mesure transcendante et les nombres «nombrés» qui sont une propriété passagère de certains ensembles (on distingue ainsi le "cinq en soi », la "pentade», et le fait qu'il $\mathrm{y}$ ait cinq personnes à une table ou cinq arbres dans un bosquet) ${ }^{16}$. Certes, pour dire qu'il y a cinq personnes à une table, il faut les compter, faire une opération mentale, mais, disent Platon, Plotin et Simon Frank, le nombre existe en lui-même.

Parlant des empiristes, Frank note:

Le mérite d'avoir réfuté dans leur principe toutes les théories de ce genre et d'avoir mis en valeur la nature du nombre, en tant que principe idéal, appartient à l'œuvre remarquable de Frege sur les fondements de l'arithmétique ${ }^{17}$. Le nombre ne peut être une propriété des choses saisie par l'expérience, car il est applicable à tous les contenus de la pensée sans exception, et il est indépendant de toute perception. Il ne peut non plus, pour la même raison, être une propriété d'un mécanisme psychique; et toutes les tentatives de déduction psychologique du nombre le présupposent d'avance et se ramènent,

13. Voir Plotin, Traité sur les nombres ("Ennéade", VI, 6[34]), Paris, J. Vrin, 1980 (trad. et commentaire par J. Bertier et al.).

14. S. Frank, La connaissance et l'être, chap. X, «Le temps et le nombre», p. 245-266; texte russe, p. 325-362 (un certain nombre de notes de bas de page n'est pas traduit en français).

15. Voir Platon, Philèbe, 15a et l'étude du père Saffrey, «L'emploi du mot "hénade" avant Proclus et chez Proclus", in Proclus, Théologie platonicienne, Paris, Les Belles Lettres, 1978, t. III, p. XI-LIIVII.

16. Voir Plotin, traité Sur les nombres, chapitres 4, 5 et 16 et l'étude d'E. Amado, «À propos des nombres nombrés et des nombres nombrants chez Plotin (Enn. VI, 2, 6)», Revue philosophique, 78, 1953, p. 423-425.

17. Note de Frank: «Frege, Die Grundlagen der Arithmetik, 1884. Ce livre est l'un des rares exemples d'ouvrage vraiment philosophiques écrits par un spécialiste des mathématiques; il est remarquable du point de vue historique, parce qu'il contient une critique péremptoire [уничтожающая критика: littéralement « une critique qui réduit à rien»] du psychologisme bien avant qu'un jugement correspondant sur le psychologisme en logique ait réussi à influer sur la littérature philosophique générale. D’où l'importance hors pair de ce livre dans l'histoire de la philosophie moderne». On peut lire l'ouvrage en français: G. Frege, Les fondements de l'arithmétique, Paris, Seuil, 1969 (trad. par C. Imbert). 
en fin de compte, au type de définition ridiculisé par Russell: «le nombre 10 est le résultat de dix actes d'attention ${ }^{18}$.

Le cas du nombre permet de comprendre l'altérité de l'âme humaine et de son «objet de savoir»: le nombre existe par lui-même, il n'est pas construit comme une interprétation, ni reçu comme un fait d'expérience. Plus encore, la «science du nombre" est une science totale au sens où connaître le nombre 10, pour reprendre l'exemple de Russell, c'est aussi connaître le nombre 9 ou le nombre 11.

Commentant le traité 9, Pierre Hadot explique ceci:

Plotin veut laisser ici entrevoir un type de réalité totalement intérieure à ellemême, c'est-à-dire dans laquelle chaque partie, en se distinguant, reste dans le tout et est en elle-même le tout dans une simultanéité parfaite, en opposition au mode de réalité propre à la rationalité et à la discursivité, dans lequel les parties se succèdent et se distinguent les unes des autres ${ }^{19}$.

Cette réalité totalement intérieure à elle-même, c'est la sagesse, la Sophia dont parle particulièrement le traité 31 Sur la beauté intelligible:

Cette sagesse, l'on en voit la grandeur et la puissance, puisqu'elle a avec elle et qu'elle a produit tous les êtres, que tous la suivent, qu'elle est elle-même les êtres et qu'ils sont nés avec elle, que les deux ne font qu'un, que, là-bas, l'être c'est la sagesse. Nous n'arrivons pas à le comprendre, parce que nous croyons que les sciences sont faites de théorèmes et d'un amas de propositions: ce qui n'est pas vrai, même dans les sciences d'ici-bas ${ }^{20}$.

La science en tant que science est une totalité-multiple qui sert de modèle à Simon Frank pour notre rapport au monde en général. Un savoir actuel n'est que la partie émergée (à la conscience) d'un savoir plus vaste et fondateur.

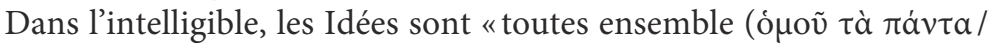
homou ta panta)» selon la formule que Frank cite au début du chapitre VI. Plotin écrit:

Ainsi donc là-bas «la vie est facile» et la vérité est pour eux leur mère, leur nourrice, leur substance et leur nourriture; ils voient tout, non pas les choses pour lesquelles il y a une génération mais celles qui sont substantielles, et ils se voient eux-mêmes parmi toutes ces choses: tout est transparent, rien d'obscur ou de résistant; chacun est clair à soi-même jusque dans son intimité, et cela est vrai pour tous; c'est la lumière pour la lumière ${ }^{21}$.

18. S. Frank, La connaissance et l'être, p. 246-247 (texte russe, p. 326-327). L'édition russe donne la référence de la citation de B. Russell: The Principles of Mathematics, I, 1903, p. 114.

19. Plotin, Traité 9, Paris, Cerf, 1994 (trad. par P. Hadot), p. 164.

20. Plotin, traité Sur la beauté intelligible, V, 8 [31], 4, 44-50, in Ennéades (trad. par É. Bréhier).

21. Ibid., lignes 1-6. 
Si la citation de la première ligne vient de l'Iliade (chant VI, vers 138 à propos de la vie des dieux), l'idée générale du passage vient du Phèdre où Platon affirme que la vérité est la nourriture de l'âme.

La vérité est la substance même de la vie de l'esprit; entendons: de même que la fausseté est un autre nom du non-être (traité De l'origine des maux, I, 8 [51]), de même la vérité est un nom de l'être. Le traité De l'éternité et du temps précise cette conception plotinienne de la vérité: «L'être est un tout véritable qui n'est pas fait d'un assemblage de parties, mais qui, afin d'être véritablement un tout, engendre ses parties. Là-bas, la vérité n'est point accord avec autre chose (ou sumphônia pros allo); elle appartient à chaque être même dont elle est la vérité ${ }^{22}$. La vérité n'est pas ici la qualité d'une proposition ou d'un raisonnement, elle n'est pas correspondance entre la parole et l'être, elle est ce qui unifie, fortifie et assure l'identité du monde intelligible (la fausseté et le mensonge, à l'opposé, divisent, opposent et provoquent la corruption). En V, 8 [31] comme en III, 7 [45], la notion d'alèthéia est associée à celles de tout et de parties. La vérité fonde l'entr'expression. Tout est dans tout («le soleil y est tous les astres, et chaque astre y est le soleil et tous les astres $")^{23}$. Une telle vision de l'intelligible a permis à Georges Rodier, dans un article de 1902, de rapprocher les Idées de Plotin et les monades de Leibniz:

Il [Plotin] conseille, pour se représenter le monde intelligible, d'imaginer une sphère transparente, qui contiendrait tous les êtres de l'univers, puis d'en abstraire l'étendue, d'en faire un tout indivisible mais qui conserve la multiplicité, où il $y$ ait une multitude d'affections et de rapports, quoiqu'il n'y en ait point de parties, dont chaque élément implique et révèle immédiatement tous les autres $[\mathrm{V}, 8,9]$. Ailleurs, il le dépeint comme une pyramide dont toutes les parties seraient animées et lumineuses, unies par leur sommet et illuminées par l'Intelligence universelle [VI, 7, 15]. [...] Plotin donne déjà de sa pensée une expression plus adéquate quand il parle de la vie universelle, de l'harmonie parfaite du monde intelligible, de ses parties dont chacune est totale [III, 2, 1]. Comme les monades de Leibniz, les Idées sont des miroirs vivants perpétuels de l'univers intelligible, des miroirs actifs indivisibles les points de vue du tout, des simplicités fécondes, des mondes en raccourci, des parties totales ${ }^{24}$.

Une telle vision de l'être et de la connaissance de l'être est très proche de ce que Frank propose dans ses deux principaux ouvrages. Reprenant la notion leibnizienne de raison suffisante, Frank note:

22. Plotin, traité De l'éternité et du temps, III, 7 [45], 4, 9-12, ibid.

23. Plotin, traité Sur la beauté intelligible, V, 8 [31], 4, 6-10, ibid.

24. G. Rodier, «Sur une des origines de la philosophie de Leibniz» [1902], in Études de philosophie grecque, Paris, J. Vrin, 1957, p. 338-351, ici p. 341. 
C'est dans cette liaison de tout contenu particulier avec l'unité où il est enraciné que consiste cette nature de la connaissance qu'on exprime parfois par la loi de la raison suffisante (закон достаточнаго основания / zakon dostatotchnavo osnovaniia) ${ }^{25}$. Cette loi exige que tout contenu distinct soit pensé comme lié à d'autres contenus; elle montre qu'un contenu particulier ne peut trouver sa justification que dans la liaison des contenus, autrement dit sur la base de l'unité pensée; pris séparément, sans rapport avec l'unité, sans lien avec son au-delà, ce contenu n'est pas encore un contenu de connaissance ${ }^{26}$.

Du même coup, le «concevable» ne prend sens que dans un horizon d' «inconcevable», notre compréhension finie dépendant d'une "notion complète» qui enveloppe l'infini. Notre connaissance par concepts ne correspond que très partiellement à la vérité de l'être. Et c'est alors vers un autre penseur, fortement influencé par Plotin, c'est vers Bergson ${ }^{27}$ que Frank se tourne:

On peut appliquer ici la célèbre comparaison de Bergson, qui assimile la pensée notionnelle [отвлеченной мысли / otvletchenoi' mysli, littéralement la pensée abstraite] à la cinématographie: celle-ci, par une série d'images discontinues, tend à reconstituer la plénitude vivante et continue de l'objet en n'atteignant jamais complètement son but ${ }^{28}$.

Seule l'intuition peut nous donner accès à cette plénitude vivante. Frank cite alors assez librement un ensemble de passages des Ennéades pour soutenir la thèse de la possibilité d'une connaissance intuitive de la totalité de la vérité:

Dans le monde d'ici-bas, une partie naît d'une autre, et chacune n'est qu'une partie; mais là (dans le monde intelligible, accessible par voie d'intuition), chaque partie découle de tout et est en même temps le tout et une partie: elle se présente comme une partie, mais se manifeste comme le tout à un œil

25. Voir G. W. Leibniz, Monadologie, $\$ 36$ et la présentation de ce «principium reddendae rationis sufficientis» dans V. Carraud, Causa sive ratio, Paris, PUF, 2002, p. 428-440.

26. S. Frank, La connaissance et l'être, p. 155-156 (texte russe, p. 206-207).

27. Voir l'ouvrage de R.-M. Mossé-Bastide, Bergson et Plotin, Paris, PUF, 1959 et l'étude de C. Rutten, «La méthode philosophique chez Bergson et chez Plotin», Revue philosophique de Louvain, 58, 1960, p. 430-452.

28. S. Frank, La connaissance et l'être, p. 236; l'image célèbre se trouve dans H. Bergson, L'évolution créatrice [1907], in Euvres, édition dite du centenaire, Paris, PUF, 1959, p. 753 (voir la conclusion: «le mécanisme de notre connaissance usuelle est de nature cinématographique»). Bergson a été traduit très vite en russe: L'évolution créatrice en 1909; l'Essai sur les données immédiates de la conscience en 1910 et Matière et mémoire en 1911. Le père Florensky cite également en bonne part l'image du cinématographe de L'évolution créatrice: voir La colonne et le fondement de la vérité [1914], Lausanne, l'Âge d'homme, 1975 (trad. par C. Andronikof), p. 307. 
perçant, pareil à l'œil de Lyncée qui pénétrait jusque dans les entrailles de la terre, selon le mythe qui fait allusion à cette vision intellectuelle $[\ldots]^{29}$.

Frank cite encore trois autres textes: II, $6,1^{30}$; III, $2,1^{31}$ et VI, 2, $3^{32}$. On le voit, en 1915, la première lecture de Frank est centrée sur la noétique plotinienne.

Dans L'inconcevable, c'est bien davantage à l'Un, le premier principe absolument transcendant que Frank se réfère. Frank est tout à fait sensible à l'écart entre Platon et Plotin et suit, sciemment ou non, le conseil que donnait Leibniz de ne pas lire Platon «ex Plotino aut Marsilio Ficino ${ }^{33}$. Il note ainsi, à la page 140 de l'édition française, que le statut des Idées est différent dans le platonisme de Platon si l'on peut dire et dans le néoplatonisme, dans un cas les Idées existent en soi, dans l'autre elles existent "dans l'esprit divin " ${ }^{34}$, autant dire dans l'Intellect hypostatique, dans la seconde hypostase. Ce point est clairement le point de désaccord entre Plotin et la présentation de la démiurgie du Timée, démiurgie selon laquelle le dieu aurait tourné son regard vers les Idées intelligibles pour fabriquer le monde. Plotin n'a de cesse de dire que le dieu intellect, le Nô̂s comme dit le grec, s'identifie aux Idées qu'il pense et que les Idées ne sont en somme que des modalités de l'Intellect divin. L'un des traités les plus éloquents sur cette doctrine est le traité $32(\mathrm{~V}, 5)$ Que les intelligibles ne sont pas en dehors de l'Intellect. Frank affirme nettement: "Il faut, en ce sens, avec Plotin - contre Platon ou le platonisme classique - reconnaître

29. S. Frank, La connaissance et l'être, p. 237 (texte russe, p. 315); Plotin, traité Sur la beauté intelligible, V, 8 [31], 4, 21-26. La traduction de Bréhier plus fidèle au texte grec est la suivante: «Ici, de chaque partie différente vient une lumière différente, et chacune est seulement une partie: là-bas, c'est du tout que vient éternellement chaque chose, et en même temps chaque chose est aussi le tout; on l'imagine bien comme une partie, mais un regard perçant y voit le tout; comme si l'on avait une vue pareille à celle de Lyncée qui, dit-on, voyait même ce qu'il y a à l'intérieur de la terre; car cette fable nous suggère l'idée des yeux tels qu'ils sont là-bas». Lyncée, l'un des Argonautes, sert également de modèle à la vision intuitive de l'esprit dans la Lettre VII de Platon (344a).

30. Plotin, traité De la qualité et de la forme, II, 6 [17], 1, 8-12: «Là, tout est un, ici, en vertu de la séparation des images, l'un est ceci, l'autre cela; de même dans le sperme tout est commun et chaque partie est tout, il n'y a ni bras, ni tête séparément; tandis qu'ici les parties s'isolent et se séparent, car elles ne sont que des images et non la réalité».

31. Plotin, traité De la Providence, III, 2 [47], 1, 31-33: «Dans le monde intelligible [...] la partie représente le tout, toutes les parties sont proches les unes des autres et ne se séparent pas, et rien ne devient purement "autre chose", isolé du reste $[\ldots]$ ».

32. Plotin, traité Sur les genres de l'être, VI, 2 [42], le chapitre 3 presque en entier.

33. G. W. Leibniz, Die philosophischen Schriften, Gerhardt (éd.), Hildesheim, Olms, 7, p. 147; signalons que c'est l'édition de Leibniz que cite Frank dans son appendice «Sur l'histoire de la preuve ontologique», Предмет знания, p. 437-504.

34. Frank dit «в божественном духе», Непостижимое, je cite l'édition parue à Moscou en 1990 aux Éditions «Правда», ісі р. 268. 
que le "monde des idées" ne peut être pensé dans son être qu'existant dans l'“esprit divin" - dans l'unité englobante qui est non pas abstraitement extratemporelle, mais concrètement supra-temporelle ${ }^{35}$. La concrétude des Idées plotiniennes permet d'ailleurs de les installer dans un monde et d'en faire des réalités vivantes et pensantes, des esprits bienheureux dans un monde de pure lumière, ce qui n'est nullement le cas chez Platon qui, rappelons-le, n'hypostasie pas l'en-soi des Idées en un monde (l'expression de «kosmos noètos» est absente des écrits de Platon $\left.{ }^{36}\right)$. Frank a une vision très juste du statut absolument transcendant de l'Un, loin du syncrétisme de Soloviev pour qui les trois hypostases de Plotin, l'Un, l'Être et l'Âme correspondraient grosso modo à la Trinité chrétienne ${ }^{37}$. Frank écrit ceci:

Le fondement, ou fondation, originaire - principe originaire qui confère sens, valeur, fondation, qui instaure le processus de fondation et, en ce sens, appelle à l'être tout le reste - il est, à l'image du Bien (agathon) de Platon, en vertu de sa puissance, de sa charge signifiante, de sa perfection, d'emblée hors du champ de l'être (epekeina tès ousias presbeia kai dunamei huperechon [au-delà de l'essence, surpassant celle-ci en dignité et en puissance $\left.{ }^{38}\right]$ ); ou encore, selon les termes de Plotin, il est ce qui assume la tâche du principe même de l'être et du fondement (ousia kai hestia [essence et foyer ${ }^{39}$ ]) de toute chose. Il est bien davantage qu'être ${ }^{40}$, il est la réalité originaire par rapport à laquelle tout être est d'emblée un être dérivé, en attente de fondation ${ }^{41}$.

Plotin a le mérite pour Frank de présenter un Premier Principe qui soit à la fois inconcevable et relié à l'homme par son inconcevabilité même: il

35. S. Frank, L'inconcevable, p. 145 (texte russe, p. 272).

36. Voir J. Laurent, «L'unicité du monde selon Platon», in La mesure de l'humain selon Platon, Paris, J. Vrin, 2002, p. 57-70.

37. Voir par exemple dans les Leçons sur la divino-humanité [1878]: «En liaison avec la doctrine du Logos, et comme développement de celle-ci, on vit apparaitre dans cette même Alexandrie la doctrine néo-platonicienne des trois hypostases divines qui réalisent le contenu absolu ou expriment d'une certaine façon le rapport de Dieu en tant qu'un avec le tout, ou en tant qu'Étant avec l'essence. Les néo-platoniciens développèrent cette doctrine indépendamment du christianisme, dont le représentant principal du néoplatonisme, Plotin, savait peu de chose [...]. Il est néanmoins parfaitement indéniable qu'il existe un lien entre la doctrine de Philon et le néoplatonisme, d'une part, et, d'autre part, le christianisme, c'est-à-dire la doctrine chrétienne de la Trinité ou du Dieu trine et un", Paris, Cerf, 1991 (trad. par B. Marchadier), p. 86-87. Sur la question de la différence entre hypostases plotiniennes et hypostases chrétiennes (les Personnes de la Trinité), voir l'ouvrage de P. Aubin, Plotin et le christianisme. Triade plotinienne et trinité chrétienne, Paris, Beauchesne, 1992.

38. Platon, République, VI, 509a.

39. Voir Plotin, V, 5 [32], 5, 18, in Ennéades.

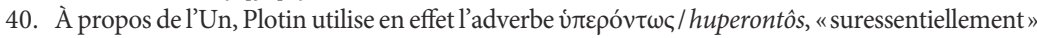
(traité 39 [VI, 8], 14, 42).

41. S. Frank, L'inconcevable, p. 352-353 (texte russe, p. 444). 
y a une trace de l'Un en nous qui nous permet de nous retourner vers la présence numineuse de l'Inconcevable. Le monde intelligible n'est qu'une étape de la conversion vers l'Un. L'unicité de chaque étant et de chaque homme en particulier est selon Frank la marque de l'Unique absolu qu'est l'Un: il y a du non-répétable, du non-conceptualisable, bref de l'absolument concret qui par sa singularité nous met sur la voie de l'Unique:

Tout "être-soi" est en même temps quelque chose d'absolument "autre", c'est-à-dire unique, non répétable, doté d'une spécificité non substituable. C'est bien pourquoi il est en un certain sens solitaire, incapable de se livrer, de s'exprimer, de se réaliser intégralement [...]. Mais c'est aussi sous ce rapport qu'il est, encore une fois, semblable et apparenté à l'Absolu lui-même - à l'unique par excellence; telle est aussi la raison de l'attirance de l'âme humaine vers l'Absolu, vers Dieu, attirance qui est - pour reprendre le mot si juste de Plotin - fuite de l'unique (solitaire) vers l'unique (solitaire) ${ }^{42}$.

Frank a tout à fait raison de donner deux adjectifs pour traduire le monos du grec, единственний / edinstvennii' et одинокий / odinokii', seul au sens d'unique pour le premier, seul au sens de solitaire pour le second. Bréhier et Fronterotta traduisent: «fuir seul vers lui seul»; Hadot: «fuir seul vers le Seul». Les traductions anglaises sont assez différentes: «the passing of solitary to solitary» ${ }^{43}$, "escape in solitude to the solitary» $44 ;$; a flight of the alone to the alone» ${ }^{45}$. Ce que Meijer appelle la «monologie» de Plotin ${ }^{46}$ est assurément une invitation à la purification et au dépouillement. La «fuite» qui est conversion de notre âme du sensible vers l'intelligible, puis de l'intelligible vers le supra-rationnel s'accomplit comme le note justement Frank selon l'antique précepte le même connaît le même: il faut une communauté de nature pour qu'il y ait rencontre. C'est donc par notre unicité que nous pouvons aller vers l'Unique. L'appel à la solitude n'est pas une promotion de la solitude d'un ermitage dans le désert, c'est plus fondamentalement la reconnaissance de ce que Frank nomme notre caractère insubstituable («неповторимое и незаменимо своеобразное»). Cette ipséité radicale met en avant l'altérité de chacun et y voit une trace

42. Ibid., p. 221 (texte russe, p. 334), Frank cite la phrase en grec après l'avoir traduite: $\varphi v \gamma \grave{~}$

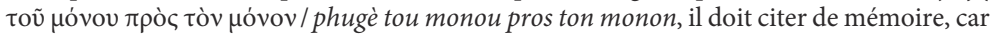

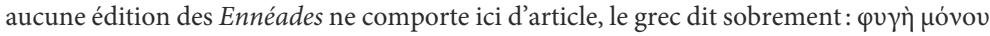

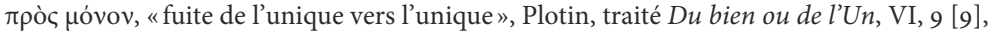
11, 50-51, in Ennéades.

43. S. Mackenna, The Enneads [1930], Londres, Penguin, 1991, p. 549.

44. A. H. Arsmstrong, Enneads, Cambridge, Harvard University Press, 1988, p. 345.

45. P. A. Meijer, Plotinus, On the Good or the One, an analytical commentary, Amsterdam,

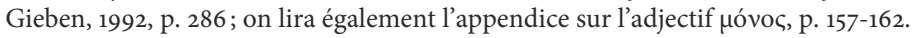

46. Ibid., p. 159. 
de l'altérité altière de l'Un; en termes plotiniens «il n'est absent de rien et il est absent de tout» ${ }^{47}$. Frank revient sur cette idée dans les dernières pages de L'inconcevable:

Cette unicité de moi-même se manifeste à son plus haut régime dans mon être avec Celui qui est, en son être même, l'Unique tout en étant, pour chacun, le Singulier, l'Autre «seulement Mien » [Особый, Иной, «только Мой»], indicible pour d'autres selon l'image qu'Il se donne pour se révéler à moi. Mon être-avec-Dieu - reprenons une fois encore les termes si justes de Plotin - est

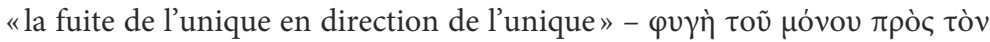
uóvov. C’est pourquoi il n'est pas de normes ni de régulations générales auxquelles il se plie. Chaque être humain a, au fond, son être-avec-Dieu particulier ${ }^{48}$.

De telles affirmations relèvent en leur fond de la pensée chrétienne et font droit au concept théologique de «Grâce» qui n'a pas d'équivalent dans la philosophie antique. L'Un de Plotin n'est pas un dieu d'amour, c'est le Premier qui, «au-delà de l'essence», est au-delà de la vie et de la pensée.

Frank lit donc Plotin à partir de sa foi chrétienne et de sa doctrine selon laquelle la philosophie de la religion ne peut ni doit se figer en dogmes généraux ou lois contraignantes. Chaque homme s'appelle à sa façon «Emmanu-el», «Dieu avec moi » ${ }^{49}$, et chaque Emmanuel a sa façon singulière d'aimer Dieu et d'être-avec-Dieu.

Une telle doctrine n'a pas cependant pour conséquence une affirmation hypertrophiée de l'individualité. L'éloge du singulier n'est pas l'apologie du solipsisme, quand chaque unique humain est en dialogue et en respiration, pourrait-on dire, avec l'Unique. Les hommes ne sont plus des entités atomiques séparées quand ils se reposent et se retournent vers un terme commun qui les aime tous, chacun pour eux-mêmes. L'adversaire de Frank, c'est une vision en quelque sorte totalitaire de l'humain, où chaque homme est interchangeable, comme des soldats sur le front de bataille, différents solo numero, seulement par leur matricule. L'affirmation de l'absolue singularité de chaque personne humaine (личность / lichnost') va de pair avec celle de l'importance décisive de la communauté. Chaque homme unique en son genre est en même temps frère des autres « uniques» dans la filiation à l'Unique. Pour appuyer cette thèse, Frank se réfère cette fois, sans les distinguer, à différents auteurs antiques:

47. Plotin, VI, 9 [9], 4, 25, in Ennéades (trad. par É. Bréhier).

48. S. Frank, L'inconcevable, p. 421 (texte russe, p. 502).

49. Ibid., p. 418 (texte russe, p. 499): «La religion chrétienne n'est pas seulement la religion de Dieu en général, mais, par-delà ce trait, elle est tout spécialement religion de Dieu en tant que mon "Père", en tant que "Dieu-avec-moi" ou "Dieu-avec-nous" (Emmanu-el)». 
La compréhension de l'être, régie par la perception de cette nature véritable du phénomène du "nous", se rencontre dans certains thèmes de la pensée antique (la doctrine d'Héraclite sur la co-appartenance et la liaison réciproque interne des consciences au moyen de leur participation au "logos" commun qui les pénètre, la doctrine des stoïciens sur le cosmos entendu comme "la cité des dieux et des hommes", la comparaison par Plotin des hommes avec les feuilles d'un arbre, séparées au-dehors, alors qu'au-dedans - par l'intermédiaire des branches entées sur le tronc commun - elles sont nourries et vivifiées par la sève commune provenant des racines); et c'est sur cette compréhension de l'être que s'appuie la doctrine mystique chrétienne, due à l'apôtre Paul, sur l'Église entendue comme le corps vivant dont les membres - les personnes dans leur distinction [отдельные люды] - se co-appartiennent intérieurement et forment une unité indivisible ${ }^{50}$.

On peut difficilement être plus syncrétique! Le logos héraclitéen, repris par les Stoïciens et par Plotin annonce le corps mystique de l'Église tel que saint Paul le présente dans la Première Épître aux Corinthiens, 12, 12-27, texte dont la postérité théologique et philosophique est considérable ${ }^{51}$. La comparaison des hommes aux feuilles d'une plante ne se trouve pas directement dans les Ennéades; le texte auquel renvoie Pierre Caussat (III, 1, 4) n'est pas tout à fait pertinent puisque l'image sert alors à représenter ce que

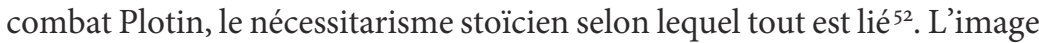
est en revanche utilisée positivement dans le traité Sur la contemplation, non pour décrire l'âme du monde et sa prétendue influence universelle (thèse stö̈cienne), mais pour faire comprendre les différents niveaux de la Procession: "Imaginez encore la vie d'un arbre immense; la vie circule à travers l'arbre tout entier; mais le principe de la vie reste immobile; il ne se dissipe pas en tout l'arbre, mais il siège dans la racine (en rhidzè hidrumenè); ce principe fournit à la plante la vie dans ses manifestations multiples; luimême reste immobile; et, n'étant pas multiple, il est le principe de cette multiplicité » ${ }^{53}$. Alors que dans le traité Du destin, l'image de la plante sert à illustrer l'immanence des parties les unes aux autres, dans le traité Sur la contemplation, c'est l'opposition et la complémentarité de la plante et des

50. Ibid., II, 6, 6, p. 274 (texte russe, p. 379-38o).

51. Sur le rapprochement de saint Paul et de la pensée stoïcienne, voir en dernier lieu, M. V. Lee, Paul, the Stoics and the Body of Christ, Cambridge, University Press, 2006.

52. Plotin, traité Du destin, III, 1 [3], 4, 1-2 et 5-9, in Ennéades (trad. par É. Bréhier) : «Est-ce une âme unique, pénétrant toutes choses, qui accomplit tout? [...] En est-il comme d'une plante, qui a son principe dans la racine, principe qui s'étend à travers toutes ses parties? Il $\mathrm{y}$ a entre elles liaison réciproque, action et passion, et, pourrait-on dire, un gouvernement unique et comme un destin de la plante (hoion heirmanenè phutou)».

53. Plotin, traité Sur la contemplation, III, 8 [30], 10, 10-14, in Énnéades (trad. de Bréhier très légèrement modifiée). 
racines qui est mise en avant ${ }^{54}$. La racine, le terme grec est au singulier, est transcendante à la plante, comme tous les jardiniers le savent, qui coupent les feuilles en attendant une repousse plus vigoureuse. C'est cette structure qu'a en vue Frank: nous sommes tous en rapport avec une énergie qui n'est pas nous, qui nous transcende et nous fonde à la fois, mais cette énergie reçue, nous étant commune, nous fonde en une communauté humaine sans pour autant abolir notre diversité. Mais le texte où il est question des «feuilles » n'est ni le traité 3 , ni le traité 30, c'est le traité 48 Sur la Providence où Plotin écrit ceci :

Le principe c'est tout en un; tout y est à la fois, chaque partie y est l'ensemble (homou panta kai holon panta); mais de ce principe, qui reste immobile en lui-même (menousè), procèdent les êtres particuliers, comme d'une racine, qui reste fixée en elle-même, provient une plante: c'est une floraison multiple où la division des êtres est chose faite, mais où chacun porte l'image du principe (eidôlon ekeinou). Mais déjà, en cette plante, certaines parties contiennent les autres; c'est que les unes sont près de la racine; les autres s'en éloignent progressivement et se subdivisent jusqu'aux ramilles du bout, aux fruits et aux feuilles (hoi karpoi kai ta phulla) ${ }^{55}$.

C'est vraisemblablement le texte auquel pense Frank quand il parle des hommes comparés "avec les feuilles d'un arbre ${ }^{56}$. L'association de Plotin avec Héraclite et les Stoïciens n'est pas due au hasard, car Plotin cite Héraclite en bonne part ${ }^{57}$ et accepte bien des arguments de la doctrine stoïcienne sur la Providence, même si, par ailleurs, il critique sévèrement l'ontologie de cette école philosophique ${ }^{58}$. Cette association permet à Frank de décaler légèrement le propos plotinien: de l'enracinement dans une puissance transcendante, il retient dans ce chapitre «Бытие “мы” / l'être du "nous" " la communauté humaine, le fait que tous les «je» ne disent jamais «je» en s'absentant de l'espèce humaine qui participe d'une façon globale à la divinité transcendante. Frank souligne que Robinson n'est pas

54. Sylvain Roux a bien montré la diversité d'utilisation de l'image de la plante, et les limites de cette image pour décrire la puissance de l'Un dans "Arbre, plante et racines», in La recherche du principe chez Platon, Aristote et Plotin, Paris, J. Vrin, 2004, p. 280-287.

55. Plotin, traité Sur la Providence (II), III, 3 [48], 7, 8-16, in Ennéades (trad. par É. Bréhier).

56. Plotin ne parle des «feuilles» que dans deux passages des Ennéades, dans le texte cité du traité 48 et dans le traité 47 pour montrer la richesse et la beauté de la variété naturelle (III, $2,13,24)$.

57. On trouve une trentaine de références à Héraclite dans les Ennéades; on notera par exemple: «Héraclite aussi connaît l'Un éternel et intelligible», V, 1 [10], 9, 3-4.

58. La reprise de la théodicée stoïcienne est particulièrement visible dans les deux traités Sur la Providence, III, 2 et 3 [47 et 48]; voir l'étude de A. Graeser, Plotinus and the Stoics, Leyde, Brill, 1972. 
seul perdu au milieu du Pacifique: «Voyez Robinson sur son île: il ne vit qu'en prenant appui sur une société et grâce aux forces d'une société» ${ }^{99}$. Et Frank précise que cet appui n'est pas dû seulement aux objets techniques récupérés du bateau, mais plus essentiellement à «la maitrise intérieure des habitudes, des compétences, des aptitudes acquises dans sa patrie, dans sa société native ${ }^{60}$. Robinson lit et écrit, c'est un animal politique doué du logos. Frank a développé dans d'autres ouvrages une pensée politique qui s'oppose fortement à l'individualisme supposé occidental ${ }^{61}$; cela, à coup sûr, ne vient pas de Plotin.

Il serait absurde de chercher un «plotinisme intégral» au XX" $X^{\mathrm{e}}$ siècle, un philosophe qui assumerait toutes les affirmations des Ennéades. Il est beau en revanche de trouver une transposition de Plotin en terre russe chez un penseur d'origine juive converti à l'orthodoxie, c'est là une bonne image de l'unicité des racines et de la pluralité de fruits.

Jérôme LAURENT

Université de Caen Basse-Normandie

59. S. Frank, L'inconcevable, p. 272 (texte russe, p. 378).

60. Ibid.

61. Voir notamment Свет во тъме / svet vo tmie: La lumière dans les ténèbres, Paris, YMCAPress, 1949. Vladimir Jankélévitch a bien noté l'importance de la communauté dans la pensée de Frank: «L'unité de l'esprit est une unité "chorale", comme l'unité "conciliaire" de la Sobornost selon Serge Troubetskoï, S. Frank et le slavophilisme russe, c'est-à-dire qu'elle repose sur l'exaltation des singularités, et non sur leur nivellement; elle ne règne pas dans le désert des multiplicités concertantes, car elle est victoire perpétuelle sur l'altérité, et non identité solitaire", Henri Bergson, Paris, PUF, 1959, p. 38. 